\title{
Ante la indiferencia: Representaciones visuales que reafirman cómo la decepción utópica se vuelve distópica
}

\author{
Hólmfríður Garðarsdóttir (Universidad de Islandia, Islandia)
}

\begin{abstract}
Every year, in an attempt to reach the United States, hundreds of thousands of undocumented migrants from Central America cross Mexico atop freight trains that are referred to by names such as "The Beast" or "The Train of Death." Driven by extreme economic conditions, civil unrest and violence in their home countries, and, in some cases, the desire to reunite with relatives already living in the United States, adult individuals, families, and even unaccompanied children and adolescents embark on this perilous journey. In doing so, they risk falling victim to abuse, extortion, sexual assault, and other forms of violence at the hands of brutal gangs, organized crime, and corrupt officials. Many lose their lives.

This study examines various aspects of the passage of undocumented Central American migrants through Mexico, viewing the situation from the perspective of human rights violations and social exclusion. It addresses the specifics and realities of the migrants' dangerous journey north, and reviews the main factors that lead these people, who are mostly from El Salvador, Guatemala, and Honduras, to leave their home countries in search of better conditions and a chance to live what they regard as the American Dream.

The experiences of Central American migrants have been the subject of several documentary films which provide both a narrative and visual representation of the journey north through Mexico. This study will analyze a series of documentaries as well as the feature films Sin nombre (2009) and La jaula de oro (2013) to consider whether the films accurately illustrate the harsh realities that undocumented migrants face while attempting to reach the United States and the extent to which they provide insight into their lives and experiences.
\end{abstract}

Keywords: Social Exclusion, Central America, Migration, Human Rights, Visual Narratives

\section{Introduction}

La atención pública internacional despertó de su indiferencia al tema del flujo migratorio de centroamericanos indocumentados por México, el día 26 de junio del 2019, al aparecer la perturbadora imagen de un padre y su hija de dos años ahogados en el río Bravo, el río que marca la frontera entre México y EEUU. ${ }^{1}$ Durante varias horas la foto circulaba en las redes. A los dos o tres días otras noticias dominaban los medios de comunicaciones.

1 Para más información véase: https://www.nytimes.com/es/2019/06/26/foto-de-migrantes-ahogados/ o https://www.bbc.com/mundo/noticias-internacional-48765026 Consultados en junio, 2019. 
Mientras tanto, desde el hemisferio norte, se consolidaba la imagen de Centroamérica como sede de inseguridad, violencia y corrupción, consecuencias, en términos socioeconómicos, de un patrón de desarrollo que produce la desigualdad y la pobreza. Resurgían noticias de inequidad e injusticia, del narcotráfico destinado a los Estados Unidos, de operaciones de pandillas delictivas como ejes operativos de criminalidad variada, incluida la trata de personas (Millett y Pérez 2005, Dudley 2010, Kovacy y Benes 2011, entre otros). ${ }^{2}$ Los causantes principales de esta inestabilidad y violencia, tanto doméstica como social, confirman ser la pobreza extrema y la falta de una proyección futura, tanto de las autoridades como del sector privado. ${ }^{3}$

Como consecuencias de tales circunstancias salen a menudo representaciones artísticas - incluidas las narrativas visuales, tanto el arte cinematográfico como el cine documental -, que representan esta realidad social y cultural conflictiva donde las condiciones de vida están fuertemente marcadas por una variedad de aspectos políticos, sociales, económicos y culturales desfavorables, particularmente para la población joven que busca modelos a seguir para desarrollarse y establecer su sentido de ser y pertenecer (Palacios Vivas 2014, Garðarsdóttir 2015 y 2016). A modo de acercamiento inicial resulta oportuno precisar que a la narrativa visual se la entiende como "el otro archivo de la historia" (Panesi 2003, 14) ya que revela contextos sociales, culturales y económicos explícitos vistos desde un punto de vista determinado. Además, las intersecciones de clase, origen, género y edad hacen que excedan fronteras socialmente reconocidas (Cooper 2015), ya que ponen en cuestión valores sociales dados por supuestos, como las responsabilidades de las autoridades por mantener el orden y proteger a los ciudadanos, además de cuestionar el papel de la familia como espacio y garantía de bienestar (More 2015). Dentro de esta gama de representaciones se encuentra un retrato de la experiencia diaria de hombres, mujeres y niños indocumentados, cuyo denominador común es la pesadilla de cruzar el territorio mexicano. Resulta por lo tanto indispensable tener presente que: "Todo migrante, a pesar de su estatus migratorio irregular, tiene el derecho a la vida; al no sometimiento a la tortura, a la esclavitud y al trabajo forzoso, a la igualdad ante la ley e igual protección de la ley y de acceso a la justicia" (ICHRP, 2010, $5-6){ }^{4}$

Según el reportaje Niñez y migración en América Central y América del Norte: Causas, políticas, prácticas y desafíos (Anónimo 2015), cada año son hasta 400.000 los centroamericanos que hacen el camino hacia el norte, a través del territorio mexicano (Varela Huerta 2017, s.p.), procediendo principalmente de Guatemala, El Salvador y Honduras (Rodríguez et al. 2011, 1). Este transcurso aumentó desde mediados de los ochenta como consecuencia de los conflictos armados en la región y la falta general de proyección futura de las diversas autoridades locales, desarrollo que continuó hasta llegar a un máximo histórico en 2005 (Caspar Olvera 2012). Sin embargo, entre 2005 y 2010 fue documentada una tendencia decreciente debido a factores como la crisis económica de EEUU y el establecimiento de

\footnotetext{
2 Para más información véase: “Desde la celda - Calle 18” (2017), en: https://www.youtube.com/watch?v=NK520ZmWBK0 y "Gangs by countries/Gangs in Mexico" https://en.wikipedia.org/wiki/Category:Gangs_in_Mexico Consultados en junio, 2019.

${ }_{3}^{3}$ Para más información se recomienda leer informes de organismos internacionales como: Banco Mundial (Anónimo 2018), Washington Office on Latin America (WOLA 2014), Comisión Económica para América Latina y el Caribe (CEPAL 2011), Comisión Interamericana de Derechos Humanos (CIDH 2013), La Agencia de la ONU para los Refugiados (ACNUR 2014), Programa de las Naciones Unidas para el Desarrollo (PNUD 2014), entre otros. También: Niñez y migración en América Central y América del Norte: Causas, políticas, prácticas y desafíos (Anónimo 2015).

${ }^{4}$ Representa: International Council of Human Rights Policy (ICHRP).
} 
mayores medidas de control migratorio, tanto en la frontera sur como en la del norte. La creciente inseguridad en México ha contribuido igualmente a este descenso (Caspar Olvera 2012, Rodríguez et al. 2011, 2). ${ }^{5}$

Por ende, este análisis — que comprende las películas Sin nombre (2009) y La jaula de oro (2013) y los documentales Fronteras al limite (2015), Coyote (2011), La Bestia (2011), Los invisibles (2010), Which Way Home (2009) y De nadie (2005) - tiene el propósito de examinar el escenario actual de los flujos migratorios desde América Central hacia los Estados Unidos centrado en la situación de tránsito para determinar cómo están representadas tales experiencias. Además, con el propósito de situar el debate en un marco teórico, se ha examinado la migración desde el contexto de los derechos humanos y la exclusión social, haciendo hincapié en la idea oportuna del sueño dorado, la utopía, según la presentó Thomas More (1516/2015), para desmantelar la realidad distópica en la cual se encuentran los migrantes durante su trayecto migratorio y/o después. Asimismo, se discute la coherencia entre la información brindada mediante los documentales por un lado y su representación cinematográfica por el otro.

\section{Las múltiples dimensiones de la exclusión}

Antes de proceder al análisis propiamente dicho, resulta oportuno destacar que la exclusión social se parte entre dos esferas principales: la económica y la social. Con la primera se hace referencia a la inaccesibilidad de participar en los sectores productivos y los comercios mercantiles y financieros, lo que se une a la insuficiencia de ingresos, la inseguridad del empleo y el desempleo o la privación material por falta de ingresos. Además, impide el aprendizaje de las habilidades para desenvolverse prósperamente en tales ámbitos (Beltrán et al. 2007, 6). En cuanto a la segunda, John Pierson mantiene que el proceso de la exclusión social - tanto cultural como política - aparece principalmente como una consecuencia de la pobreza o de los bajos ingresos, al mismo tiempo considera que factores como la discriminación social y de género, junto a niveles bajos de educación y de niveles de vida son componentes que juegan un papel determinante en el proceso $(2003,7)$.

Las formas de exclusión, entonces, entendidas como "desempoderamiento extremo que, si no son neutralizados por el acceso a la ciudadanía social, desembocan en situaciones de no participación en dinámicas básicas de pertenencia en la sociedad" (Pérez Sainz et al. 2006, 11-12), puestas en evidencia en la narrativa visual estudiada, son multidimensionales e implican el rechazo, total o parcial, de la participación plena en la vida de una sociedad determinada. Para ejemplificar mejor la exclusión tan omnipresente en el caso de los migrantes centroamericanos resulta apropiada la siguiente definición presentada por la Organización Mundial de la Salud (OMS):

Exclusion consists of dynamic, multi-dimensional processes driven by unequal power relationships interacting across four main dimensions - economic, political, social and cultural - and at different levels including individual, household, group community,

\footnotetext{
5 Para más información véase: 5 libros recientes para entender a la Centroamérica de hoy“. En línea: www.laprensa.com.ni/2018/05/22/cultura/2423105-5-libros-recientes-para-entender-la-centroamerica-de-hoy, del 22 de mayo 2018, consultado en junio de 2019.

${ }^{6}$ Para estudiar con más detalle la situación de la mujer migrante se recomiendan, entre otros, los documentales Los feminicidios del Estado de México (2015), Feminicidios (2011), Femicidio/Feminicidio - Ni una más (2011) y Killer's Paradise (2006). Véase también Los Invisibles (2012, min. 7.30- 8.30 y 10.29-11.05).
} 
country and global levels. It results in a continuum of inclusion / exclusion characterized by unequal access to resources, capabilities and rights (en Escorel et al. 2008, 2).

Dicho de otro modo, la exclusión es un proceso que priva a los individuos, las familias y/o los grupos de los recursos necesarios para participar en las actividades culturales, sociales, económicas y políticas. De manera similar, las prácticas discriminatorias que se producen pueden estar profundamente arraigadas en la comunidad, la familia, las instituciones públicas, etc., tanto como en la idiosincrasia de un pueblo expresada por las normas y valores ejercidos. De modo comparable, Behrman et al. definen el concepto como "la negación de acceso igual a las oportunidades impuesta por ciertos grupos de la sociedad sobre los demás" $(2003,11){ }^{7}$

Por otra parte, la exclusión manifiesta la interacción de una variedad de circunstancias, acontecimientos y procedimientos que afectan a los individuos o grupos, negándoles la posibilidad de alcanzar una calidad de vida digna (Beltrán et al. 2007, 5-6). Las diferentes dimensiones de la exclusión incluyen entonces no sólo límites en la participación activa en el mercado laboral, sino también la negación o limitación sistemática del acceso a los recursos y los servicios sociales, por ende, la pobreza, la falta de voz política, de protección social, de acceso a la justicia y la negación del derecho a condiciones de igualdad en las relaciones sociales (GSDRC, "social exclusion as a process"). Consecuentemente, la sociedad excluyente sería aquella en la que los individuos se relacionan e interactúan principalmente con otros del mismo grupo o clase social, y aquella en la cual las interacciones condicionantes por raza o clase son clave para acceder a puestos de trabajo, crédito, oportunidades de educación y opciones de atención médica (Behrman 2003, 12). ${ }^{8}$

Debido a lo anteriormente dicho, el proceso de la exclusión social y por ende la marginalización, en forma de desventajas y discriminación, no se limita a los niveles más bajos de la jerarquía social, sino que pueden manifestarse independientemente de la pobreza en niveles sociales distintos. En cierto modo, según Pérez Sainz et al., la exclusión social es un problema estructural de los estados, refiriéndose a la ineficiencia de las políticas públicas al abordarla en toda su magnitud. Es decir, la ambición de reducir la pobreza ha sido ineficaz y el mercado, cuyas fallas son estructurales, no ha ofrecido salidas de superación de la exclusión social, sino que, al contrario, ha tendido a reproducirla (2006, 214). Asimismo, la exclusión termina en la negación de una identidad social y cultural propia, además de cualquier tipo de espacios de recreación. La privación de estos elementos puede tener efectos negativos en el desarrollo de habilidades y aptitudes dentro de entornos como la comunidad local (Beltrán et al. 2007, 6). Es interesante para el estudio aquí presentado comprobar que las condiciones de pobreza comúnmente engloban dimensiones espaciales y que los grupos excluidos a menudo se concentran en zonas vulnerables como los barrios o villas de emergencia o áreas periféricas de baja inversión donde faltan oportunidades. Estas áreas segregadas de los entornos urbanos carecen de centros de salud y escuelas y cuando existen son muy precarios. Es en este entorno donde irremediablemente emergen altos niveles de criminalidad, violencia, miseria, delincuencia y drogadicción.

Finalmente, al hablar de exclusión social se alude también a la falta del acceso pleno a los derechos civiles, políticos y humanos. Esto se evidencia en la ausencia de participación

\footnotetext{
${ }^{7}$ Cabe destacar que la exclusión social es un término para el cual no existe una definición única y consensuada: "Se originó en Francia en la década de los setenta para describir la condición de ciertos grupos al margen de la sociedad que fueron cortados tanto de fuentes regulares de empleo y las redes de seguridad de los ingresos del Estado del bienestar" (Pierson 2003, 4).

${ }^{8}$ En inglés dice: "The denial of equal access to opportunities imposed by certain groups of society upon others".
} 
política, la representatividad limitada o ineficiente y la falta de poder en la toma de decisiones que afectan a la vida cotidiana de las personas (Beltrán et al. 2007, 6). Según explican Behrman et al., una de las formas más evidentes de las desigualdades sociales es la exclusión institucionalizada, que se revela por medio de la negación de una voz y representación en las decisiones públicas que sufren los grupos excluidos. Siguen encontrando barreras a la hora de buscar la participación activa, el acceso a la educación al empleo en el sector formal y la igualdad de sueldo, además de enfrentarse con obstáculos judiciales. En este caso, pese a que existe la igualdad jurídica formal, las mujeres, junto con otras minorías, a menudo se enfrentan a la discriminación (OHCHR "What are human rights") ya que la legislación está prejuiciada contra ellas. De este modo, tener igualdad ante la ley no significa la ausencia de la exclusión (Behrman 2003, 12).

\section{La ilusión utópica como promotor de la migración}

Al trasladar la atención al fenómeno de la migración indocumentada por México, se observa que las causas de la misma es una combinación de factores: exclusión económica y/o social, condiciones precarias marcadas por la pobreza y la violencia, además de la existencia de familiares ya establecidos en los EEUU. Desafortunadamente estas condiciones no parecen que vayan a cambiar ya que la Federación Internacional de Derechos Humanos (FIDH 2008) afirma que durante la última década la pobreza no ha dejado de aumentar en las zonas rurales y semiurbanas de los países del triángulo norte. ${ }^{9}$ Como consecuencia, y mientras muchas familias dependen de las remesas de familiares que ya están trabajando en EEUU, sobre todo en las zonas rurales y periféricas (51), numerosos jóvenes deciden emprender el viaje promovidos por la esperanza o, quizás, la ilusión de "mejorar las condiciones en que viven" sus familiares, como aparece revelado en el documental Los invisibles (min. 3:20), aunque reconocen que el proceso transitorio consiste en hacer desplazamientos en rutas migratorias complejas y peligrosas.

Sin entrar a discutir las condiciones actuales de la zona fronteriza entre México y EEUU, los cruces irregulares de migrantes centroamericanos hacia los EEUU se producen mayoritariamente en la frontera con Guatemala (véase: La jaula de oro, 2013 y De nadie, 2005). Allí ya se encuentran con varios obstáculos como son los secuestros, asaltos, violaciones, extorsiones y la trata de personas. ${ }^{10}$ A partir de la entrada a México el medio de transporte que ha pasado a representar visualmente la migración indocumentada hacia los EEUU son los trenes de carga comúnmente llamados "de la muerte" o "la Bestia" (véase: Fronteras al límite, 2015 y La Bestia, 2011). Para facilitar el trayecto, numerosos migrantes utilizan los "polleros" o "coyotes" (véase: Coyote, 2011), quienes a menudo se dedican a extorsionar y a secuestrar migrantes (Rodríguez et al. 2011, 6).

Al acercarnos al estudio de la narrativa visual elegida observamos que la película Sin nombre (Cary Fukunaga, México, 2009) presenta un retrato de la experiencia común de

\footnotetext{
${ }^{9}$ Según la FIDH, 19 millones de los 35 millones de habitantes de los países centroamericanos (Guatemala 18 millones, Honduras 10 y El Salvador 7 millones) viven en la pobreza, de los cuales 8 millones se consideran en pobreza extrema (FIDH 2008, 9). Simultáneamente, en "Poverty in Central America: Advancements and Needs", se confirma que: "The poorest $20 \%$ of the population receive only $3 \%$ of all income; the wealthiest $20 \%$ receive $60 \%$ " (IFAD 2016, s.p.).

${ }^{10}$ La presentación de la historia de María (véase: De nadie 2005) revela el abuso sexual sufrido por las migrantes y las consecuencias culturales que implican que no pueda volver a casa por la vergüenza y la discriminación social de haber sido violada.
} 
hombres, mujeres y jóvenes al cruzar el territorio mexicano. ${ }^{11}$ Desde el comienzo Fukunaga introduce a su protagonista, una adolescente hondureña, Sayra (Paulina Gaitán) quien se reúne con su padre que ha sido expulsado de EEUU y que quiere volver a entrar. A Sayra entonces se le presenta la opción de emprender el viaje por México junto a él. Ya dentro del territorio mexicano conoce a Casper (Edgar Flores) alias Willy, un adolescente mexicano miembro de la pandilla Mara Salvatrucha, MS-13. Casper acaba de ingresar a un nuevo candidato a miembro de la banda, un niño de doce años, Smiley (Kristyan Ferrer), después de que éste pase por un proceso de iniciación violenta (min 3.25-4.30). Aun así, la posición de Willy dentro de la pandilla peligra ya que han descubierto que tiene una novia fuera de la banda (min. 25.25). Después de que maten a su novia (min. 34.15), Willy se enfrenta al jefe de la Mara, Lil Mago (Tenoch Huerta Mejía), ya que abusa constantemente de los indocumentados. Finalmente, Willy mata al jefe y decide abandonar la pandilla para convertirse en uno más de los indocumentados (min. 41.20).

La película muestra las condiciones en las que viajan los protagonistas - además de muchos otros viajeros -, mostrando una pauta utilizada en los documentales analizados, como es el caso de La Bestia (2001). En él, Clara y Noel, hermanos guatemaltecos, hablan de asaltos y ladrones que los violaron y robaron en la frontera. José y Omar de Honduras fueron víctimas de robos de pandillas que les amenazaron con machetes y pistolas. Mientras, otros reportan robos y extorsiones por parte de la policía, un hecho que el general Ramos, jefe de la Policía de Arriaga, verifica como acontecimiento diario y declara que la corrupción es como un cáncer presente en todas las corporaciones del que nadie puede escapar. Alejandro Solalinde, sacerdote y director de un Albergue para Migrantes en Chiapas, también critica las autoridades afirmando que:

El problema de todos los que caen en el tren, que los matan, que los asaltan, es que de muchos de ellos nunca se sabe y nunca se sabrá [...] van a la fosa común. Es increíble que en México, que firma tantos tratados contra la tortura [...] que estamos viviendo estas cosas tan terribles (min. 49.28-50.01).

En el documental, así como en la película Sin nombre (2009), se revela cómo la amenaza se vuelve omnipresente y cómo la desconfianza da origen a la falta de solidaridad tan necesaria para sobrevivir. No obstante, entre los protagonistas de la película se perciben momentos de inseguridad y miedo, pero también de tranquilidad y esperanza. Encuentran la oportunidad de compartir expectativas personales (min. 56.50-57.30 y 1.23.30), aunque resulte indispensable contemplar a la vez los peligros y las inseguridades, representación atestiguada en todos los documentales. Mientras Fukunaga intercala numerosas escenas mostrando la belleza de México y la bondad del pueblo mexicano al ofrecer comida y bebidas a los viajeros, en el trayecto hacia el norte todos tienen que aguantar la persecución de las pandillas, las confrontaciones con las autoridades, locales y nacionales, el hambre, la caída de colegas del tren, entre otras atrocidades. ${ }^{12}$ El espectador atestigua momentos de alegría y amistad en los personajes de los Sin nombre, antes de volverse testigo de la matanza del padre de Sayra y la captura de su hermano, el escape de Casper y Sayra de la Mara (min. 1.18.40) y

\footnotetext{
${ }^{11}$ La película fue ganadora del premio "Mejor Dirección" y "Excelencia en Fotografía” en el Festival de Sundance 2009.

${ }^{12}$ La Federación Internacional de Derechos Humanos (FIDH) reporta que hay impunidad total frente a las violaciones cometidas en contra de los derechos humanos de los migrantes, como se revela en el hecho de que casi no existen casos de condena judicial, que se traduce en la falta de voluntad política de alcanzar soluciones para proteger a los migrantes en tránsito $(2008,38)$.
} 
su descanso de tres días en un albergue antes de intentar cruzar la frontera por medio de un "coyote". Más tarde observa la captura de Casper a orillas del río y su ejecución por Smiley al proclamar con determinación "Mara por vida" (min. 1.26.50). Seguidamente, la narración termina con un close-up de Sayra, después de lograr cruzar el Río Grande, al llamar al número que le había pasado su padre (min 1.30.30).

Otro artefacto representativo del escenario actual de los flujos migratorios desde América Central hacia los Estados Unidos, y que sirve para evaluar la credibilidad del arte cinematográfico, se encuentra en la película La jaula de oro (Diego Quemada-Diez, México, 2013). La película nos introduce a tres protagonistas jóvenes, Juan (Brandon López), Sara (Karen Martínez) y Samuel (Carlos Chajon), todos menores de edad, que comienzan el tránsito desde Guatemala (véase también: La Bestía min. 6.11 y Coyote min. 22.25). Su destino es la ciudad de Los Ángeles, California. No obstante, estando todavía en el sur de México, en Chiapas, las autoridades los capturan y los devuelven. Después de estudiar sus opciones, Juan y Sara deciden intentar el viaje de nuevo mientras Samuel decide que no. Se enreda el viaje de los dos al acercarse el joven indígena Tzozil, Chauk (Rodolfo Domínguez). Chauk no habla casi español y Sara, que se hace pasar por un chico, se vuelve el eje de la comunicación - el intérprete entre los tres. Las amenazas continuas los llevan a sus límites y prueban sus principios mientras, simultáneamente, tienen que cuidar su integridad y luchar por su vida (véase también: Coyote min. 28.10-40.00 y Los invisibles min. 1.38-3.46). Tanto como en Sin nombre (2009) el medio de viaje es el tren de carga, "la Bestia" y a medida que avanza el viaje el tren se vuelve el símbolo tanto de los peligros que tienen que confrontar como seres humanos y sujetos sociales indocumentados, como la representación de la esperanza de alcanzar el paraíso del norte y por ende un bienestar utópico.

Una vez más el espectador se encuentra confrontado con innumerables escenas violentas y el paso de los días marcados por la inseguridad omnipresente. La temática enfocada por Quemada-Diez encuentra un paradigma en el hilo conductor del documental Which Way Home (2009), ${ }^{13}$ donde aparecen entrevistados varios niños y adolescentes migrantes. El documental se centra en Kevin y Fito, hondureños de catorce años. Kevin espera comprarle una casa a su madre para que pueda salir de una relación abusiva y Fito, quien ha vivido con su abuela en condiciones precarias, espera que le adopte alguna familia al otro lado de la frontera. A pesar de las pruebas de valor, perseverancia y agilidad, cada testimonio ostenta los peligros y los riesgos a que se enfrentan los niños. Aquí, como en el otro material consultado se enfatiza la vulnerabilidad extrema causada por la edad y el género, además de la desesperación causada por la falta de experiencia (véase: Fronteras al límite 2015, min. 27.25-30.10 y La Bestía 2011, min. 12.18). Tanto en la película La jaula de oro, como en la mayoría de los documentales, se examinan las causas que obligan a los jóvenes salir de los países del triángulo norte que parecen haber consolidado un patrón de desarrollo que produce y reproduce la desigualdad y la pobreza. Todos reportan carecer de oportunidades económicas y a pesar de su minoría de edad, se sienten obligados a ayudar a sus familias. Su visión ilusoria está marcada por una meta poco relevante, pero "muy clara" (Los invisibles, min. 20.20) y por "cumplir el sueño o morir en el intento", como lo explica Silvia en De Nadie (min. 49.15). Como consecuencia, mientras al comienzo del trayecto reina el optimismo y deseo de "seguir adelante" (Los invisibles min. 20.31) y se expresa esperanza de que todo saldrá bien al cantar: "Un año no es un siglo y yo volveré" (Coyote min. 12.50), el ir y venir de los indocumentados confirma la observación del narrador de Los invisibles al

\footnotetext{
${ }^{13}$ La película ganó el premio “Outstanding Information Programming”, Emmy 2010.
} 
revelar que continuamente se enfrentan a una realidad dominada por un ambiente donde el "orden no permite al 'otro'" (min. 19.41).

\section{Conclusiones}

En resumidas cuentas, se consideran a las narrativas visuales estudiadas como testimonios y archivos verosímiles sociales, que ofrecen representaciones fidedignas del viaje migratorio. Su denominador común es revelar las condiciones persistentes de exclusión y por ende la necesidad de abandonar su país de origen por la falta absoluta de esperanza por un futuro próspero. Los migrantes indocumentados son más vulnerables a la violencia ejercida por las autoridades, tanto locales como nacionales, y por el crimen organizado, que controla las rutas migratorias. Están sometidos a la exclusión económica por ser pobres y a la social por la constante violación de sus derechos básicos. Son víctimas en tanto que la autoridad, que tiene el deber de protegerlos, los trata de forma indigna y no les ofrece protección oficial tras haber sufrido abusos por parte del crimen organizado, además de otros episodios de corte racista y xenófobo.

La narrativa analizada confirma por lo tanto que, para acercarse a la complejidad del fenómeno migratorio los cineastas de los documentales se han puesto en contacto directo con los migrantes y su realidad. Los entrevistados dan testimonio de sucesos de gran crueldad; todos cuentan el mismo relato. Una historia de gente marginada, que sale de sus países en búsqueda de una calidad de vida mejor, pero que sigue siendo explotada allá a donde vaya. Sus testimonios sirven luego como materia prima para el arte cinematográfico como las películas Sin nombre (2009) y La Jaula de Oro (2013), ya que resultan ser relatos realistas del viaje migratorio, en donde los protagonistas se enfrentan con los mismos obstáculos, riesgos y abusos montando en "la Bestia" que los migrantes entrevistados en los documentales. Se confirma pues que los migrantes son presa fácil ya que sufren una discriminación múltiple: en sus países de origen, a través de la ausencia de una vida digna, y en los países receptores, de tránsito o destino, donde frecuentemente se les niega el acceso a sus derechos civiles y humanos. Como aparece revelado en el material estudiado, el tránsito por el estado mexicano significa la violación de los derechos fundamentales de estos individuos, como en la mayoría de los casos no pueden contar con el apoyo o protección de autoridades estatales y civiles. La corrupción se desvela en la participación de la policía y las instituciones de migración con el crimen organizado. Esta ausencia por parte de los organismos responsables de cumplir con las leyes y los tratados internacionales implica la discriminación sistemática y la exclusión, tanto económica como social, de este grupo social.

Ante esta indiferencia y a pesar de que los migrantes declaren que el paso por México es un infierno, las narraciones fílmicas no condenan a todos los mexicanos como culpables de dicha discriminación. Al contrario, dirigen su crítica hacia las autoridades estatales y municipales por la corrupción tan arraigada y por no cumplir con su rol de proteger los derechos humanos de un grupo social marginado y vulnerable al abuso. Por medio de la narrativa visual analizada, los múltiples testimonios de delitos y violaciones representan situaciones de discriminación sistemática en el sentido de que las victimas están excluidas al acceso a tratamiento digno y a la justicia, cuya responsabilidad está en manos de la autoridad. Cualquier ausencia de protección implica una violación a los tratados internacionales de los derechos humanos. Dichas condiciones son confirmadas por Héctor García, de la Comisión de Derechos Humanos (La Bestia, 2011), que admite que las autoridades siguen abusando de los 
migrantes, aunque mantiene que las cifras han bajado. Concluye con que el discurso de Derechos Humanos se concentra en una esfera estéril [...] en las vías, en los techos del tren, en las escalerillas de los vagones no hay evidencia real de que ese discurso existe y actúa.

Los protagonistas de las dos películas, así como los entrevistados en los documentales, empiezan el camino con el mismo sueño, marcado por ideas ambiguas de cómo es la sociedad "del norte" y de lo que harán al llegar. Su versión comúnmente representa una esperanza ilusoria que no tiene correlación con la realidad, ya que viajan sin tener una noción realista del viaje por México ni de la estancia en los Estados Unidos. El carácter representativo de las narrativas visuales estudiadas confirma en definitiva las múltiples formas de exclusión social, la vulnerabilidad del migrante y la absoluta falta de resoluciones políticas. Tanto las películas como los documentales son una demostración de las realidades "distópicas" personales y sociales de los migrantes indocumentados.

\section{Bibliografía}

ACNUR. 2014. "Niños en fuga: niños no acompañados que huyen de Centroamérica y México y la necesidad de protección internacional." La Agencia de ONU para los Refugiados. ACNUR. Consultado en junio, 2019. http://www.acnur.org/t3/fileadmin/scripts/doc.php?file=t3/fileadmin/Documentos/Publ icaciones/2014/9568.

Anónimo. 2015. Niñez y migración en América Central y América del Norte: Causas políticas, prácticas y desafíos. Consultado en junio, 2019. http://cgrs.uchastings.edu/sites/default/files/NinezMigracionDerechosHumanos_Espa \%C3\%B1ol_1.pdf

Anónimo. 2018. "El Banco Mundial en América Latina y el Caribe". Consultado en enero, 2018. http://www.bancomundial.org/es/region/lac

Behrman, Jere., Gaviria, Alejandro. Székely, Miguel. eds. 2003. "Social Exclusion in Latin America. Perception, reality and implications." Who's in and Who's out: Social exclusion in Latin America. Washington D.C: Interamerican Development Bank.

Beltrán, María A., Cruz, José M. y Savenije, Win. 2007. "Exclusión social, jóvenes y pandillas en Centroamérica." $1^{\text {a }}$ ed. Fundación Dr. Guillermo Manuel Ungo. $\begin{array}{llll}\text { FUNDAUNGO. Consultado junio, } & 2019 .\end{array}$ http://fundaungo.org.sv/pdf/2012/temas_acualidad/Temas $\% 20 \mathrm{de} \% 20$ Actualidad $\% 20 \mathrm{~N}$ o.\%203.pdf.

Caspar Olvera, Selene. 2012. "Migración México-Estados Unidos en cifras (1990-2011)". Red Internacional de Migración y Desarrollo. Consultado en junio, 2019. http://www.scielo.org.mx/scielo.php?script=sci_arttext\&pid=S1870759920120001000 $\underline{04}$

CEPAL. 2011. "Migración internacional en América Latina y el Caribe: nuevas tendencias, nuevos enfoques." Comisión Económica para América Latina y el Caribe. CEPAL, Consultado en junio, 2019. http://www.cepal.org/es/publicaciones/migracioninternacional-en-america-latina-y-el-caribe-nuevas-tendencias-nuevos. 
CIDH. 2013. "Derechos humanos de los migrantes y otras personas en el contexto de la movilidad humana en México." Comisión Interamericana de Derechos Humanos. CIDH. Consultado en junio, 2019.

http://www.oas.org/es/cidh/migrantes/docs/pdf/informe-migrantes-mexico-2013.pdf

Cooper, Brittney. 2015. "Intersectionality". The Oxford Handbook on Feminist Theory. Lisa Disch y Mary Hawkesworth (eds.). Oxford: Oxford Handbooks Online.

Dudley, Steven S. 2010. Drug Trafficing Organizations in Central America: Transportistas,Mexican Cartels and Maras. Washington D.C.: Woodrow Wilson Insternational Center for Scholars.

Escorel, S., Hernández, M., Johnston, H., Methieson, J., Popay, J. y Rispel, L. 2008. "Understanding and tackling Social Exclusion: Final Report to the WHO Commission on Social Determinants of Health From the Social Exclusion Knowledge Network." World Health Organization. WHO. Constuldado en junio, 2019. http://www.who.int/social_determinants/knowledge_networks/final_reports/sekn_final \%20report_042008.pdf.

FIDH. 2008. "Muros, abusos y muertos en las fronteras: Violaciones flagrantes de los derechos humanos de los migrantes indocumentados en camino a Estados Unidos." Consultado en junio, 2019. https://www.fidh.org/es/region/americas/estadosunidos/Muros-abusos-y-muertos-en-las-fronteras.pdf

Garðarsdóttir, Hólmfríður. 2015. "El cine centroamericano y la feminización de la vulnerabilidad”. Pobreza, globalización y violencia en el cine latinoamericano del siglo XXI. Santiago, Chile: Cuarto Propio, pp. 157-181.

Garðarsdóttir, Hólmfríður. 2016. "Creando subjetividades a través del arte cinematográfico. Historias sobre transformación personal en el cine centroamericano de los últimos años". Revista Latina de Sociología, Facultad de Ciencias Sociales, Universidad de Coruña, España, s.p.

GSDRC. Falta año. "Social exclusion". Governance and Social Development ResourceCentre. GSDRC. Consultado en junio, 2019. http://www.gsdrc.org/go/topicguides/social-exclusion.

GSDRC. Falta año. "Social exclusion as a process". Governance and Social Development Resource Centre. GSDRC, s.f. Consultado en junio, 2019. http://www.gsdrc.org/go/topic-guides/social-exclusion as a process.

IFAD. 2016. "Strategy for rural poverty reduction in Latin America and the Caribbean." IFAD, s.p. Consultado en junio, 2019.

https://www.ifad.org/where/region/resource/tags/pl/1962687

ICHRP. 2010. Migración Irregular, Tráfico Ilícito de Migrantes y Derechos Humanos: Hacia la Coherencia. Constulado en junio, 2019.

www.ichrp.org/files/summaries/40/122_pb_es.pdf

OHCHR. Falta año. "What are Human Rights." Oficina de Alto Comisionado para los Derechos Humanos. OHCHR. Consultado en junio, 2019. http://www.ohchr.org/SP/Issues/Pages/WhatareHumanRights.aspx.

Kovacy, Sara P. y Alexander N. Benes (eds.). 2011. Central America. Security Challenges, New York: Nova Science Publishers, Inc. More, Thomas. 2015. Utopía. Trad. Emilio García Estébanez. Madrid, España: Akal. 
Millett, Richard L. y Orlando J. Pérez. 2005. "New Threats and Old Dilemmas: Central America's Armed Forces in the 21st Century". Journal of Political and Military Sociology, vol. 31, no. 1, 59-80.

Palacios Vivas, Nydia. 2014. Escritoras ejerciendo la palabra. Una mirada crítica nicaraguense. Managua, Nicaragua: 400 Elefantes.

Panesi, Jorge. 2003. "Villa, el médico de la memoria." Archivos de la memoria. Ed. Ana María Barrenechea. Rosario, Argentina: Beatriz Vitebro Editora, pp. 13-25.

Pérez Sainz, Juan P., Salas y Minor Mora. 2006. "De la pobreza a la exclusión social. La persistencia de la miseria en Centroamérica." Fundación Carolina. Fundación Carolina San José. Consultado en junio, 2019. http://www.fundacioncarolina.es/wpcontent/uploads/2014/07/Avance_Investigacion_6 .pdf.

Pierson, John. 2003. Tackling Social Exclusion. London: Routledge.

PNUD. 2014. "La pobreza en El Salvador: una mirada de sus protagonistas." Programa de las Naciones Unidas para Desarrollo. PNUD. Consultado en junio, 2019. http://www.sv.undp.org/content/dam/el_salvador/docs/povred/UNDP_SV_Miradas_P obreza_2015.pdf

Rodríguez et al. 2011. "Apuntes sobre migración. Migración centroamericana de transito irregular por México". Centro de estudios migratorios del INM. Consultado en junio, 2019. http://oxfammexico.org/wp/content/uploads/2013/06/APUNTES_N_Julio2011.pdf

Varela Huerta, Amarela. 2017. "La trinidad perversa de la que huyen las fugitivas centroamericanas: violencia feminicida, violencia de estado y violencia de mercado". Consultado en junio, 2019. https://www.sciencedirect.com/science/article/pii/S0188947817300063\#!

WOLA. 2014. La otra frontera de México. Seguridad, migración y la crisis humanitaria en la línea Centroamérica. Isacson, Adam, Maureen Mayer y Gabriela Morales (eds.). Consultado en junio, 2019. http://www.wola.org/sites/default/files/La\%20otra\%20frontera.pdf

\section{Narrativas visuales}

Cammisa, Rebecca. 2009. Which Way Home. EEUU, falta ciudad: Mr. Mudd y HBO Films.

Dirdamal, Tin. 2005. De nadie. México D.F.: Producciones Tranvía.

Fukunaga, Cary. 2009. Sin nombre. México D.F.: Creando Films y Canadá Films.

Jiménez Pons, Susana. 2011. Feminicidio S.A. Madrid: Televisión española.

Loyola, Bernardo. 2015. Los feminicidios del Estado de México. Falta ciudad: Vise News Productions.

Rodríguez, Chema. 2011. Coyote. Madrid: Televisión española.

Portenier, Giselle. 2006. Killer's paradise. Canada, falta ciudad: BBC.

Silva, Jaime. 2015. Fronteras al límite. La frontera de la Bestia. Madrid: Televisión española.

Silver, Mark y Gael García Bernal. 2010. Los invisibles. México D.F.: Greenhouse studios.

Ultreras, Pedro. 2011. La Bestia. Falta ciudad: Coproducción entre México, EEUU, El Salvador y Guatemala. 
Quemada-Diez, Diego. 2013. La jaula de oro. México D.F.: Coproducción Machete Productions.

Díaz Madrigal, Yunuen y Martin Sabio. 2011. Femicidio/Feminicidio - Ni una más. Falta ciudad: Bleu video. 